\title{
BICEP2, Planck, spinorial space-time, pre-Big Bang
}

\section{On the possible origin of primordial CMB B-modes and gravitational waves. Potentialities of alternative cosmologies and open questions}

\author{
Luis Gonzalez-Mestres ${ }^{1, a}$ \\ ${ }^{1}$ Megatrend Cosmology Laboratory, Megatrend University, Belgrade and Paris \\ Goce Delceva 8, 11070 Novi Beograd, Serbia
}

\begin{abstract}
The field of Cosmology is currently undergoing a positive and constructive crisis. Controversies concerning inflation are not really new. But after the 2013-2014 Planck and BICEP2 announcements, and the more recent joint analysis by Planck, BICEP2 and the Keck Array (PBKA), the basic issues can involve more direct links between the Mathematical Physics aspects of cosmological patterns and the interpretation of experimental results. Open questions and new ideas on the foundations of Cosmology can emerge, while future experimental and observational programs look very promising. The BICEP2 result reporting an excess of $B$-mode polarization signal of the cosmic microwave background $(\mathrm{CMB})$ radiation was initially presented as a signature of primordial gravitational waves from cosmic inflation. But polarized dust emission can be at the origin of such a signal, and the evidence claimed by BICEP2 is no longer secure after the PBKA analysis. Furthermore, even assuming that significant $\mathrm{CMB} B$-mode polarization has indeed been generated by the early Universe, its theoretical and cosmological interpretation would be far from obvious. Inflationary gravitational waves are not the only possible source of primordial CMB $B$-modes. Alternative cosmologies such as pre-Big Bang patterns and the spinorial space-time (SST) we introduced in 1996-97 can naturally produce this polarization. Furthermore, the SST automatically generates for each comoving observer a local privileged space direction (PSD) whose existence may have been confirmed by Planck data. If such a PSD exists, vector perturbations have most likely been strong in the early Universe and may have produced CMB $B$-modes. Pre-Big Bang cosmologies can also generate gravitational waves in the early Universe without inflation. After briefly describing detectors devoted to the study of the CMB polarization, we discuss the situation emerging from BICEP2 results, Planck results and the PBKA analysis. In particular, we further analyze possible alternatives to the inflationary interpretation of a primordial $B$-mode polarization of cosmic microwave background radiation.
\end{abstract}

\section{Introduction}

How can Cosmology explore the ultimate mathematical structure of space-time, and what are the implications of different cosmological approaches on the polarization of cosmic microwave background radiation? The two questions appear closely related after the 2013-2014 Planck and BICEP2 results

\footnotetext{
ae-mail: luis.gonzalez-mestres@ cosmology.megatrend.edu.rs
} 
and the controversies that have followed these announcements. The original BICEP2 preprints $[1,2]$ appeared in the context of a pre-existing debate $[3,4]$ on the predictions of inflationary models $[5,6]$ that followed the 2013 Planck results [7-9]. The possible generation of primordial gravitational waves leading to $\mathrm{CMB} B$-modes, as a consequence of cosmic inflation, was precisely a crucial issue.

However, the presence of such an experimental signature in BICEP2 data was never certain [10], as the observed signal could actually be due to galactic dust effects [11, 12]. The joint Planck-BICEP2 - Keck Array (PBKA) analysis [13,14] has found no conclusive evidence for the primordial CMB $B$-modes initially announced. Instead, an upper limit has been obtained on the possible contribution from inflationary gravitational waves. The tensor-to-scalar ratio turns out to be $r_{0.005}<0.12$ at $95 \%$ confidence level with a scalar pivot scale of $0.05 \mathrm{Mpc}^{-1}$.

It is widely recognized that the experimental program of which BICEP2 is a component looks very promising in all cases $[15,16]$ and will help to clarify fundamental questions in Cosmology. But instrumentation and data analysis and interpretation are difficult tasks. Furthermore, as already underlined in [17] and later in [18], if the experimental and phenomenological uncertainty remains and requires a long-term effort, the situation is not different from a theoretical point of view [19].

The standard cosmological model (Big Bang + inflation $+\Lambda \mathrm{CDM}$ ) is far from being well-defined and well-established. In particular, it cannot completely account for recent cosmological observations and analyses [20-22]. It can also be challenged from a more fundamental point of view including space-time structure and the formation of the Universe[18, 23].

Alternative cosmologies [24, 25] must be seriously taken into account and can be more performing to explain data than the standard pattern based on the conventional Big Bang with inflation.

\subsection{Theoretical uncertainties}

The initial discrepancies on the cosmological consequences of inflation triggered by Planck results have been followed by controversies [26, 27] around the interpretation of BICEP2 data, leading even to the strong assertion "the inflationary paradigm is fundamentally untestable, and hence scientifically meaningless" [28]. The debate on this subject is actually not new. Doubts on the reasonableness of using inflation as a mainstay of modern Cosmology had already been emitted previously [29, 30].

In any case, and even if the PBKA analysis has by no means ruled out cosmic inflation, it seems reasonable to conclude that the study of the "very early" Universe is just beginning.

Not only long-term observational and experimental programs are clearly required, but theory appears to need a similar effort. In particular, the validity of the approach based on General Relativity with equations dominated by a cosmological constant (the standard $\Lambda$ ) and some form of cold dark matter (CDM) remains an open question [23, 24] and alternative scenarios can be considered [37].

Indeed, assuming that some experimental data will eventually turn out to correspond to a primordial $B$-mode polarization of cosmic microwave background radiation, the theoretical interpretation of such a signal would be far from obvious $[17,18]$ if the analysis is not constrained a priori by the standard cosmological assumptions, including the mathematical space-time structure.

The initial BICEP2 result was often presented as a strong direct evidence for cosmic inflation and primordial gravitational waves, arguing that: i) the $B$-modes of CMB cannot be generated primordially by scalar (density) perturbations; ii) only gravitational waves (tensor perturbations) generated by the inflationary expansion of the Universe can produce this polarization. But such a reasoning ignored primordial vector perturbations as a possible source of CMB $B$-modes, as well as alternative ways to generate primordial gravitational waves without inflation. Possible vector perturbations (vorticity) are not really considered in this respect within the conventional cosmological framework, as they are assumed to have been damped by inflation [31]. However, this analysis applies only to standard cosmologies based on the conventional Big Bang approach where inflation is a basic ingredient. 
Alternative cosmologies without inflation [32] can naturally incorporate efficient primordial vector perturbations. They can also generate primordial gravitational waves potentially leading to observable signatures in suitable present-day detectors. The graviton and all the standard "elementary" particles may also turn out to be composite objects generated by a more fundamental pre-Big Bang evolution [33]. Then, other primordial waves may have existed associated to the ultimate constituents of matter. It is even not excluded that remnant free preons and preonic waves still exist in our Universe.

\subsection{Alternative cosmologies}

Alternative cosmologies can naturally lead to a completely different situation from that usually considered in the inflationary interpretation of a possible signature of primordial CMB $B$-modes:

- Pre-Big Bang models [23, 25] do not in general involve an inflationary scenario, and are in principle able to produce primordial CMB $B$-modes through vector perturbations $[17,18]$ leading to observable signals. They can naturally solve the horizon problem [32], and provide sensible alternatives to the inflationary description of the formation of conventional matter structure in our Universe.

- The spinorial space-time (SST) we suggested in 1996-97 [34, 35] automatically generates [33, 36] a privileged space direction (PSD) for each comoving observer [21, 37]. Then, the possible existence of primordial CMB $B$-modes can appear as a natural consequence of this intrinsic local space anisotropy of geometric and cosmic origin. The existence of the PSD, already compatible with WMAP data [38], may have been experimentally confirmed by more recent Planck results [20]. The PSD combined with parity violation can potentially explain the observed CMB anisotropy that involves an asymmetry between the two hemispheres defined by a preferred direction [21, 24].

- Pre-Big Bang models can also generate primordial gravitational waves without the standard cosmic inflation, as explicitly shown in an original approach based on an initial gravitational instanton at cosmic time $t=0[39,40]$. More fundamental waves may also have existed in this kind of pre-Big Bang pattern that allows for further interesting developments. In other pre-Big Bang models, incorporating for instance new ultimate constituents of matter or different forms of pre-matter, the generation of primordial gravitational waves together with the graviton and the rest of standard particles is in general a natural phenomenon assuming that standard particles are the dominant vacuum excitations.

Alternative cosmologies, including pre-Big Bang scenarios based on the SST, have not been excluded by observational and experimental data. New physics beyond the Planck scale, or even at larger distance scales and lower energies, can be a natural extension of standard theories if quantum mechanics ceases to hold or undergoes modifications at very high energy and very small distance scales [23, 41]. Similarly, the effective space-time structure can depend on the energy or distance scale $[32,36]$, or even on the matter present in the space-time region considered $[18,19]$.

More than a century after the prediction of gravitational waves by Henri Poincaré in 1905 [42], such waves have not yet been found experimentally but they play an increasing role in modern Cosmology. Similarly, Albert Einstein considered as early as 1920 [43] possible deviations from relativity at very short distance scales. Later, quantum field theory appeared to be perfectly compatible with Lorentz symmetry. But in the recent period, data at higher energies have become available. As a result, possible violations of relativity and of other standard fundamental principles of Physics, including quantum mechanics and conservation laws, are now a promising field of research [36, 41].

In this contribution, we further develop the analysis of [17] on possible alternatives to the inflationary interpretation of experimental results that would confirm the detection of $B$-modes of the CMB polarization corresponding to a signal from the early Universe. We also comment on some recent Planck data and discuss their possible cosmological implications. In view of the present situation, we consider some unconventional approaches to Particle Physics and Cosmology (including patterns where the Planck scale does not make sense [41]), and analyze their potentialities. 


\section{BICEP2 and related experiments}

The BICEP2 experiment is a component of a larger and very ambitious program installed at the South Pole and devoted to the experimental study of the polarization of the cosmic microwave background radiation. Although the basic goal of this program is to check the validity of the inflationary scenario, other important uses of such an experimental effort should also naturally emerge.

BICEP (Background Imaging of Cosmic Extragalactic Polarization), also called the Robinson Gravitational Wave Background Telescope and now called BICEP1, was the name of an experiment deployed to the Amundsen-Scott South Pole Station in November 2005 [44]. The experiment was designed specifically to measure the $B$-mode polarization of CMB radiation at degree angular scales, having already in mind to test cosmic inflation through the detection of the gravitational wave background on the CMB polarization. The signature provided by primordial CMB $B$-modes was thought to be predicted only by inflationary models, as alternative cosmologies were not considered.

The Robinson Telescope [45] is a refractive telescope with a 250-mm aperture providing an instantaneous field-of-view of 17 degrees with angular resolution of 55 arcminutes at $100 \mathrm{GHz}$ and 37 arcminutes at $150 \mathrm{GHz}$. It was made of fourty-nine pairs of horn-coupled polarization-sensitive bolometers. These bolometers were cooled to $250 \mathrm{mK}$ using a ${ }^{4} \mathrm{He} /{ }^{3} \mathrm{He} /{ }^{3} \mathrm{He}$ sorption fridge system with a ${ }^{3} \mathrm{He}$ InterCooler and a ${ }^{3} \mathrm{He}$ UltraCooler. The results of its three sessions of operation (20062008) have been analyzed in [46] and contain no possible evidence for primordial CMB $B$-modes. The BICEP experiment (BICEP1) has been the beginning of a long-term program incorporating progressively improved detectors with an evolving technological approach.

After BICEP1, an improved detector (BICEP2 [2]) was installed and operated in 2010-2012. BICEP2 has a focal plane of 512 antenna coupled transition edge sensor (TES) bolometers involving 512 sensors (256 pixels) at $150 \mathrm{GHz}(\simeq 2 \mathrm{~mm}$ wavelength) with a SQUID readout. Each pixel's antenna is $7.2 \mathrm{~mm}$ on side, and each TES bolometer is made of $\mathrm{Ti}\left(\mathrm{T}_{c} \sim 520 \mathrm{mK}\right)$ and $\mathrm{Al}\left(\mathrm{T}_{c} \sim 1.34\right.$ $\mathrm{K})$ connected in series. The focal plane is cooled to $270 \mathrm{mK}$, reaching a sensitivity of $87.2 \mathrm{nK}$ per square-degree pixel $(5.2 \mu \mathrm{K}$. arcminute) over an effective area of 384 square degrees.

BICEP2 has been designed to measure CMB polarization on angular scales of 1 to 5 degrees with $\ell=40-200$, where $\ell$ stands for the angular multipole. The BICEP2 team explicitly presents the experiment as "built to search for the inflationary gravitational wave background through B-mode polarization on angular scales around $2^{\circ} "$ [2]. But the same set of experiments briefly described here can play an important role in the exploration of possible alternative cosmologies.

From a technological point of view, BICEP2 has been the first experiment to use the antennacoupled TES arrays [47-50] fabricated at the Jet Propulsion Laboratory (JPL) [51]. The TES technology, based on the strong temperature dependence of resistance in the phase transition of a superconductor ( $\mathrm{Ti}$ and $\mathrm{Al}$ in the BICEP2 sensors), allows in particular for a more dense setup with arrays incorporating a much larger number of pixels than in the case of spider-web bolometers.

Close to the BICEP2 experiment, the Keck Array [52] uses the same technology. It was completed in 2012 [53] and modified in 2013-2014 [49]. The Keck array is made of five polarimeters, each with a design similar to that of BICEP2 but with a pulse tube refrigerator instead of the $\mathrm{He}_{4}$ Dewar. The Keck Array contains 2560 detectors at 150 and $100 \mathrm{GHz}$.

After BICEP2, BICEP3 [54] is an improved refracting telescope with an aperture of $550 \mathrm{~mm}$, a focal plane area 5 times larger than BICEP2, a faster optics and 1280 dual-polarization pixels (2560 bolometric detectors). Designed for CMB polarimetry at $95 \mathrm{GHz}$, it is expected to be able to better reject background due to galactic dust in the observation of primordial CMB $B$-modes.

Similarly, a new polarization sensitive receiver, SPT-3G, has been designed [55] for the South Pole Telescope with a high signal-to-noise allowing to go beyond simple statistical detection of $B$-mode CMB lensing and hopefully be able to separate lensing and primordial $B$-modes. 
POLAR [56, 57] is a long-term project. Polar-1 involves about 5000 bolometric detectors at 150 $\mathrm{GHz}$. At a later stage, the Polar Array should contain ten such telescopes, leading to the most sensitive cosmic microwave background polarimeter in operation.

\subsection{Other experiments}

In parallel, POLARBEAR [58] has published results in the $500<\ell<2100$ range rejecting at $97.2 \%$ confidence level the hypothesis of no $B$-mode polarization power from gravitational lensing. An effective sky area of 25 square degrees was observed, with 3.5 arcmin resolution at $150 \mathrm{GHz}$. The POLARBEAR experiment, based in the Atacama desert in the north of Chile at an altitude close to $5.200 \mathrm{~m}$, utilizes a unique focal plane of 1,274 antenna-coupled, polarization sensitive TES bolometers (637 pixels) cooled to 250 milliKelvin [59]. POLARBEAR-2 will feature a larger area focal plane, with 7,588 TES bolometers [60].

The Atacama Cosmology Telescope (ACT) [61], designed to map CMB over a large sky area with an angular resolution of 1 arcmin, designed to observe at frequencies around 148, 218 and $277 \mathrm{GHz}$. Equipped with a new polarization sensitive receiver, ACTpol [62] and observing at $146 \mathrm{GHZ}$, ACT has detected [63] gravitational lensing of the CMB polarization by large-scale structure at a statistical significance of $4.1 \sigma$ from polarization data and at $9.1 \sigma$ combining temperature and polarization data. A B-mode polarization lensing signal is reported with a significance of $3.2 \sigma$.

The polarization-sensitive receiver SPTpol [64], installed on the South Pole Telescope, has measured [65] the CMB gravitational lensing potential with a signal-to-noise ratio greater than one for $100<\ell<250$. The hypothesis of no lensing is rejected at $5.8 \sigma$ from polarization data, and at $14 \sigma$ using temperature and polarization data.

CLASS (Cosmology Large Angular Scale Surveyor) [66, 67] will also operate from the Atacama Desert. It is expected to map the CMB polarization at angular scales larger than a few degrees, observing over $65 \%$ of the sky at 38, 93, 148 and $217 \mathrm{GHz}$.

SPIDER $[68,69]$ is a balloon-borne instrument designed to probe the possible primordial gravitational wave signal by detecting CMB $B$-modes at degree angular scales. It involves 2400 pairs of polarization-sensitive bolometers in the $94 \mathrm{GHz}$ and $150 \mathrm{GHz}$ frequency bands and map $7.5 \%$ of the sky with a depth of 11 to $14 \mu \mathrm{K}$. arcminute and a signal/noise ratio better than that of Planck. Again, the possible detection of primordial $B$-modes of the cosmic microwave background radiation can instead be an evidence for primordial vector perturbations in alternative cosmologies, or for both phenomena (vector perturbations and gravitational waves) simultaneously.

Another balloon project in this domain is PILOT (Polarized Instrument for the Long-wavelength Observations of the Tenuous ISM) [70, 71], whose goal is to characterize the polarization of the dust continuum emission in the diffuse interstellar medium.

Clearly, such a set of experimental programs, together with Planck and other experimental and observational devices and projects, should be accompanied by a parallel long-term effort in the domain of theory. It seems in particular compelling to explore all sensible cosmologies and elaborate relevant phenomenology to suitably interpret old and new data.

\section{BICEP2 and Planck results}

Aiming to measure the primordial polarization of the cosmic microwave background radiation and search for a possible signal of inflationary gravitational waves, BICEP2 has made public results of its 3 -year run. In March 2014, the BICEP2 Collaboration announced [1] an excess of $B$-mode power over the base lensed- $\Lambda \mathrm{CDM}$ expectation in the range $30<\ell<150$, inconsistent with the null hypothesis at a significance of $>5 \sigma$. These new data still required further analyses. 
The June 2014 version of the BICEP2 paper states that the observed $B$-mode power spectrum is well reproduced by a lensed- $\Lambda \mathrm{CDM}+$ tensor model with a tensor-to-scalar ratio $r$ of $0.20(+0.07$, $-0.05)$. The value $r=0$ is thus disfavored at $7.0 \sigma$. But such a confidence level is actually questioned by a warning on the possible contribution of foreground dust: accounting for dust effects "will shift this value downward by an amount which will be better constrained with upcoming data sets". It is explicitly admitted that models of polarized dust emission are not constrained enough to be able to exclude the possibility that this emission explains the entire observed signal.

In September 2014, the Planck Collaboration [10] reported an analysis of HFI polarization data from 100 to $353 \mathrm{GHz}$ to determine the dust angular power spectra over the range $40<\ell<600$. The result for $40<\ell<120$ turned out to be of the same magnitude as the BICEP2 signal.

In order to reduce uncertainties and assess the dust contribution to the CMB $B$-modes measured by BICEP2, the two collaborations have performed, together with the Keck Array, the already mentioned joint analysis $[13,14]$ excluding evidence for primordial CMB $B$-modes.

A previous attempt by Mortonson and Seljak to perform a similar analysis can be found in [15]. These authors obtained strong constraints on inflation favoring solutions without gravity waves, and can even possibly exclude a tensor-to-scalar ratio $r>0.14$ with $99.5 \%$ confidence.

The analyses presented in [14] and [15] following the standard cosmological approach set upper limits on the generation of primordial gravitational waves in inflationary models, but do not exclude such a phenomenon. Further experimental work is therefore required. But a more basic question concerns the dependence of the published data analyses on the cosmological approach chosen a priori $($ Big Bang + inflation $+\Lambda \mathrm{CDM})$ ignoring, in particular, possible vector perturbations.

\subsection{Other Planck results}

The first cosmological results of Planck based on measurements of the CMB temperature and lensingpotential power spectra were presented in [8]. Constraints on inflation were discussed in [9].

In January 2014, further analysing data on CMB anisotropy, Planck has also confirmed [20] the observation of an asymmetry in power between two cosmic hemispheres defined by a preferred direction. Possible signatures of parity violation (oscillations between odd and even modes) are equally present in the hemisphere where power is larger. These results make more explicit the asymmetry already found by Eriksen et al. [72] and Hansen et al. [73] using WMAP data. The power spectrum calculated by these authors for a hemisphere centred at Galactic longitude and latitude $(l, b)=\left(237^{\circ}\right.$,$20^{\circ}$ ) and over the multipole range $20<\ell<40$ turned out to be larger than in the opposite hemisphere. A similar hemispherical asymmetry had also been found by Park [74].

In March 2014, a new version of [8] has equally confirmed the observation of an "anomaly" in the multipole range $20<\ell<40$ as compared to standard $\Lambda$ CDM predictions. Such an "anomaly" is recognized to be "a real feature" of the anisotropies of primordial CMB.

On 21 March 2013, when Planck made public the first version of [20], the ESA-Planck News site wrote in an article for large public [75] : "... because precision of Planck's map is so high, it also made it possible to reveal some peculiar unexplained features that may well require new physics to be understood". Since then, the Planck Collaboration has always considered that its results favour the standard cosmological pattern except for "anomalies" possibly related to new physics.

But why should this new physics not generate an alternative cosmology able to reproduce in a consistent way the whole set of Planck results, including those usually explained through standard cosmological patterns of the Big Bang + inflation + $\Lambda$ CDM type? If new physics has been present in the early Universe, it may actually have dominated its dynamics and remain nowadays a fundamental component of the deep structure of matter and of cosmic evolution. 


\section{Cosmology, vacuum and the mathematical space-time}

Cosmological patterns are usually based on the standard space-time with four real variables and conventional relativity. The vacuum structure is described in terms of standard quantum field theory (SQFT), even if such a description leads to the cosmological constant problem. General relativity is an essential ingredient of this approach and provides the framework of standard cosmology.

A description of space-time as felt by macroscopic "ordinary" matter around us and used in standard particle physics is thus extrapolated : i) to cosmic level, including large cosmological scales ; ii) to the ultimate structure of matter, assumed to be accounted for by SQFT.

However, standard relativity can be basically a phenomenological description of space-time as seen by conventional matter at low energy in the part of the Universe it occupies [35, 92], while the global geometric and physical structure of the Universe can present a deeper configuration [24, 33] and obey different laws. The situation would then be similar for the SQFT vacuum structure [23, 36], assuming the notion of vacuum can be really well-defined on theoretical grounds [19].

The actual size of the whole Universe remains unknown, and there is by now no evidence that conventional matter is present everywhere or that standard particles are the ultimate constituents of matter. It is therefore not excluded, in particular, that other regions of the Universe be occupied by free preons or by composite forms of matter different from our standard particles [35, 41].

Similarly, the effective vacuum structure and properties can in principle vary between different regions of the Universe as seen at the largest scale in the present epoch. Even assuming an initial common origin for the whole Universe within a global pre-Big Bang era, different local vacuum configurations may have been formed in the subsequent evolution.

The structure of vacuum can in any case differ from SQFT at very small distance and time scales $[23,24]$. If new physics is present at these scales, it is expected to have played a crucial role in the formation of the very large scale structure of the Universe and the validity of General Relativity at large cosmic scales is no longer a natural property. The notion of Planck scale can also cease to hold.

\subsection{Lorentz-like symmetries and stable particle kinematics}

In all cases, metrics of the Lorentz type can remain the naturally stable kinematics for particle-like objects $[23,76]$. Writing for a particle kinematics:

$$
E^{2}+\alpha p^{2}=E_{0}^{2}
$$

where $E$ is the energy, $p$ the momentum modulus, $E_{0}$ the (positive) energy of the particle at rest and $\alpha$ a constant with the dimensions of a speed, it can be readily checked that if $\alpha>0$ the vacuum can spontaneously emit pairs of particles with $p=E_{0} \alpha^{-1 / 2}$ and opposite momenta. Therefore, the initial vacuum is unstable and a new fundamental state (the actual physical vacuum) is expected to emerge leading to a new metric for free particles with a new, negative value of $\alpha$. The situation is similar writing, for instance:

$$
E^{2}+\sum_{i=1}^{3} \alpha_{i} p_{i}^{2}=E_{0}^{2}
$$

with $i=1,2$ or 3 standing for three orthogonal space directions. If $\alpha_{1}>0$, the same vacuum instability is generated even if $\alpha_{2}$ and $\alpha_{3}$ have negative values.

It then follows [80, 81] that Lorentz-like metrics of the form (1) with $\alpha<0$ are the naturally stable kinematics for free particles. If several kinds of particles exist with different critical speeds, those with the lowest value of $-\alpha$ (the square of the critical speed) will be the naturally stable ones $[33,76]$ as the cost in energy is lower for a given value of momentum. 
The same competition occurs between light and phonons in a solid where the speed of sound is much lower than the speed of light and, in most cases, light is prevented from propagating and absorbed by the solid. But such a solid structure is not present everywhere, and light is in general allowed to propagate around us. Analogies inspired by this situation can also be considered in Cosmology if several families of free particles exist with different critical speeds in vacuum and, in particular, if the actual Universe is much larger than the one currently studied.

Then, rather than an intrinsic fundamental property of space and time, the conventional relativity of standard matter can be a low-energy symmetry similar to the effective Lorentz-like symmetry of the kinematics of low-momentum phonons in a solid lattice $[33,76]$ where the speed of sound plays the role of the critical speed. The speed of light would be the low-energy critical speed of a family of vacuum excitations (the standard particles) not directly associated to a basic space-time geometry at a deeper level. The local structure of vacuum would then be a crucial issue.

\subsection{Beyond the standard space-time}

What can then be the global geometric structure of such a Universe?

If the present structure of vacuum and the relevant Lorentz-like symmetry for particle kinematics can vary between different regions of the Universe, and/or if these symmetries are only approximate, a basic common ingredient is in any case expected to be the $\mathrm{SU}(2)$ covering group of the $\mathrm{SO}(3)$ space rotations (leaving aside the PSD at an initial stage).

It actually turns out $[25,36]$ that a simple and natural way to introduce new phenomena, beyond and at a more fundamental level than standard cosmology, can be to consider a spinorial space-time as the fundamental framework to describe the evolution of the Universe [34, 35].

It is well known that particles with half-integer spin do not feel space exactly in the same way as particles with integer spin or macroscopic objects described in the $h \rightarrow 0$ limit. As a 360 degrees rotation changes the sign of a spin-1/2 wave function, fermions do not belong to representations of the $\mathrm{SO}(3)$ group. To explore all possible consequences of this property, we introduced $[34,35]$ a spinorial SU(2) space-time with two complex coordinates replacing the four standard real ones.

The properties of the SST, including some possible cosmological and field-theoretical implications, have been reminded and further studied in [23,36] and in [21, 37]. The SST appears to be a well-suited and powerful tool when used to define cosmic coordinates, and leads to interesting natural predictions concerning the expansion of the Universe and the automatic generation of a local PSD.

Because of its complex coordinates, the SST can also be the natural space-time structure to generate quantum mechanics, as discussed with more detail in [19].

\section{The spinorial space-time (SST)}

Using the SST instead of the conventional real space-time has important implications for both the internal properties of standard elementary particles and the very large scale structure of the Universe $[18,25]$. The two domains appear directly related through a possible pre-Big Bang evolution where the ultimate structure of matter and space-time would play a leading role and dominate the overall dynamics and global geometry of the primordial Universe. It then seems natural to explore the possibility that the SST allows to explain the large-angle anomalies observed by Planck.

In this new space-time geometry, each point of the cosmic space-time is described by a SU(2) spinor $\xi$ involving two complex coordinates that replace the four standard real ones.

It is then possible, in such a spinorial formulation of the space-time geometry, to associate to $\xi$ a positive $\mathrm{SU}(2)$ scalar $|\xi|^{2}=\xi^{\dagger} \xi$ (the dagger stands for hermitic conjugate). A definition of the 
cosmic time (the age of the Universe) can then be $t=|\xi|$ with an associated space given by the $S^{3}$ hypersphere $|\xi|=t$ that carries and additional cosmic spinorial structure from the SST. Other definitions of the cosmic time $t$ in terms of $|\xi|$ (f.i. $t=|\xi|^{2}$ ) lead to similar cosmological results as long as a single-valued function is used for $t$ in terms of $|\xi|$.

With the definition $t=|\xi|$ for the cosmic time, if $\xi_{0}$ is the observer position on the $|\xi|=t_{0}$ hypersphere, space translations inside this spinorial hypersphere are described by $\mathrm{SU}(2)$ transformations acting on the cosmic spinor space, i.e. $\xi=U \xi_{0}$ with:

$$
U=\exp \left(i / 2 t_{0}^{-1} \vec{\sigma} \cdot \overrightarrow{\mathbf{x}}\right) \equiv U(\overrightarrow{\mathbf{x}})
$$

where $\vec{\sigma}$ is the vector formed by the usual Pauli matrices and the vector $\overrightarrow{\mathbf{x}}$ the spatial position (in time units, at that stage) of $\xi$ with respect to $\xi_{0}$ at constant time $t_{0}$. The antipodal point to $\xi_{0}$ in the global Universe is obtained through a $2 \pi$ rotation of this cosmic spinor. As the ratio $t_{0}^{-1} x$ naturally defines a $\mathrm{SU}(2)$ rotation angle, the translation (3) turns out to be associated to a global rotation at all values of $t$ in a similar way to a comoving distance.

The origin of cosmic time $t=0$ is then associated to the beginning of the Universe. Such an initial singularity, and possible pre-Big Bang origin, corresponds to the spinor $\xi=0$ where the initial space is contracted to a single point. This simple description of cosmic space-time leads to an expanding universe where cosmological comoving frames can be associated to straight lines going through the origin $\xi=0$. Then, the SST geometry naturally suggests the existence of a local privileged rest frame for each comoving observer, which is compatible with existing cosmological observations.

It was already pointed out in [35] that an attempt to associate to the cosmic spinor $\xi$ real cosmic space coordinates through the vector $\overrightarrow{\mathbf{x}}_{c}$ defined by the natural contraction $\overrightarrow{\mathbf{x}_{c}}=\xi^{\dagger} \vec{\sigma} \xi$ does not actually lead to such coordinates. Instead, one gets $|\xi|^{2}$ times a unit vector defining the local privileged space direction. The standard space coordinates can only be defined using an origin $\xi_{0}$ at a given cosmic time $t_{0}$ as in (3). Strictly speaking, field theory should use spinorial space-time coordinates. The implications of the SST for quantum field theory deserve being studied in detail.

Using the SST local space coordinates, space rotations with respect to a fixed point $\xi_{0}$ are given by $\mathrm{SU}(2)$ transformations acting on the spatial position vector $\overrightarrow{\mathbf{x}}$ defined by (3). A spatial rotation around $\xi_{0}$ corresponds to a SU(2) element $U(\overrightarrow{\mathbf{y}})$ turning $U(\overrightarrow{\mathbf{x}})$ into $U(\overrightarrow{\mathbf{y}}) U(\overrightarrow{\mathbf{x}}) U(\overrightarrow{\mathbf{y}})^{\dagger}$. The vector $\overrightarrow{\mathbf{y}}$, related to $U(\overrightarrow{\mathbf{y}})$ in a similar way to (3), provides the rotation axis and angle. If a spin-1/2 particle is present at the position $\overrightarrow{\mathbf{x}}$ with an associated spinor $\xi_{p}$ describing its spin, then $\xi_{p}$ transforms into $\xi_{p}^{\prime}=U(\overrightarrow{\mathbf{y}}) \xi_{p}$.

\subsection{Direct cosmological implications}

The SST automatically generates in a purely geometric way $[23,36]$, without standard relativity and without any explicit presence of conventional matter:

i) The standard relation between relative velocities and distances at cosmic scale, with a ratio $H$ (velocity/distance) equal to the inverse of the age of the Universe $\left(H=t^{-1}\right)$. Thus, the SST naturally anticipates [33, 36] the Lundmark - Lemaître - Hubble (LLH) law [77-79] usually obtained from cosmological equations incorporating standard matter and general relativity.

ii) The privileged space direction (PSD) for each comoving observer, possibly seen by Planck.

Furthermore, space translations in the SST form a (non-abelian) compact group, contrary to the standard space-time geometry of special relativity, and space rotations are described by the same $\mathrm{SU}(2)$ group. Translations are SU(2) transformations acting on cosmic coordinates, whereas rotations are $\mathrm{SU}(2)$ transformations of local coordinates leaving invariant a spatial origin $\xi_{0}$. Such a structure is therefore different from that of the standard real space-time where the Poincare group leads to no-go theorems for the unification of internal and space-time symmetries. 
As previously reminded, the PSD associated to the cosmic space-time point $\xi$ is defined by the linear combination of sigma matrices (with real coefficients) that leaves $\xi$ invariant $[23,36]$. Then, the space-time points lying on the trajectory generated by this sigma-like matrix satisfy the relation $\xi^{\prime}=\exp (i \phi) \xi$ where $\phi$ is a real phase and $\exp (i \phi)$ a complex phase factor. Such a definition of the PSD is stable under SU(2) transformations and comoving time evolution.

Thus, the existence of the PSD is an intrinsic cosmological property specific to the spinorial structure of the global cosmic space-time as "seen" from the cosmic origin $\xi=0(t=0)$ through the SST description at a deeper level than the standard space-time variables. The PSD does not violate invariance under cosmic $\mathrm{SU}(2)$ rotations, but these rotations are the equivalent of translations in our standard language. General Relativity does not incorporate such a phenomenon.

The PSD is not automatically apparent in the space-time geometry when standard space coordinates (the above $\overrightarrow{\mathbf{x}}$ ) are used, as these coordinates belong to a vector representation of SU(2) and $\mathrm{SO}(3)$. Therefore, conventional cosmology based on the usual real space-time cannot in principle account for the PSD in a simple way. We expect bosons and macroscopic objects to be less directly concerned by PSD effects than the elementary fermions, the possible ultimate constituents of matter and the very large scale structure of the Universe. The signature of a preferred direction possibly found by Planck [20], if due to the local PSD of the spinorial space-time combined with parity violation $[23,36]$ would have been generated by the specific dynamics of the early Universe.

Other cosmic evidences for the spinorial space-time and the PSD can exist, not only in BICEP2 and Planck data but more generally in observable cosmological phenomena.

\subsection{SST and CMB B-modes}

Contrary to the standard isotropic description of the early Universe, where only $E$-modes associated to gradients are assumed to be present in the primordial CMB polarization except for the $B$-modes due to inflationary gravitational waves, a cosmology based on the spinorial space-time naturally leads to primordial CMB $B$-modes generated by rotations around the local privileged space direction and vector products by this direction $[17,18]$.

As the existence of a local PSD is a permanent property of the SST and the standard inflationary expansion of the Universe is not required in pre-Big Bang models incorporating the SST geometry, we do not expect in general primordial vector perturbations to be damped as assumed in [31]. CMB $B$-modes can therefore be generated by such vector perturbations. The situation would be similar with other alternative space-time geometries.

Then, cosmic inflation and primordial gravitational waves are no longer necessary to account for the possible existence of primordial CMB $B$-modes. On the contrary, such a result, together with recent Planck data, may have provided a signature of the SST cosmic geometry or of some other unconventional structure beyond the standard space-time and cosmology.

Simultaneously, the SST geometry providing the space-time structure of a pre-Big Bang pattern is perfectly compatible with primordial gravitational waves that may have been generated in the early Universe together with vector perturbations. Then, both primordial sources may have contributed to the $B$-mode polarization of the cosmic microwave background radiation.

\subsection{Other consequences of the SST approach}

The existence of the PSD can be at the origin of a violation of local rotation invariance in Particle Physics, thus potentially invalidating the standard conservation law for angular momentum in phenomena sensitive to the PSD. Such an effect can be very difficult to detect in Particle Physics experiments, as the conventional orbital angular momentum is defined using position and momentum 
operators that are vector representations of the space symmetry group. But, like the early Universe, the internal structure of fermions may be sensitive to the PSD and generate some observable signatures.

In the above described SST approach, the global size of the Universe is given (up to a constant) by the cosmic time $t$. The expansion of the Universe is therefore part of the fundamental space-time geometry and does not appear to be directly related to the concept of energy in the conventional sense, even if a new fundamental dynamics may be implicitly at work. One can then consider that standard energy conservation does no longer follow as an exact basic law of Physics.

Although possible effects of energy non conservation due to the Universe expansion are expected to be too small to be detected in laboratory experiments, the evolution of vacuum structure and particle properties at cosmological scales deserves careful exploration.

Describing the internal structure of standard elementary fermions (quarks and leptons) through a spinorial wave function leads to a local SST with origin at the particle space-time position. For a comoving particle at $\xi_{0}$, the local spinorial coordinates of a point $\xi$ would be given by the spinor $\xi_{L}=$ $\xi-\xi_{0}$. A wave function of the type $\Psi\left(\xi_{L}\right)$ describing the lepton and quark internal structure $[23,36]$ in a SST based at $\xi_{0}$ provides an unconventional alternative to standard causality at very small distance and local time scales, as most of the values of $\xi$ thus considered do not correspond to the cosmic time $\left|\xi_{0}\right|$. At these very small scales, the notion of time itself should be reconsidered.

Assuming that the internal wave function of a standard "elementary" particle is an eigenstate of the spinorial SU(2) group and that spin corresponds to an internal spinorial angular momentum, the allowed spin values would be multiples of $1 / 2$, including $0,1 / 2,1,3 / 2$ and 2 but also possibly higher spins contrary to conventional assumptions. All particles of the standard model can be generated by a spinorial wave function, and the existence of "elementary" spin-3/2 particles seems then natural. As the standard Poincaré group is no longer an exact symmetry, an alternative to supersymmetry involving both space-time and internal symmetries may emerge as a new, in practice approximate and broken, symmetry escaping usual no-go theorems [23,36]. The subject clearly requires further study.

The experimental search for signatures of "elementary" (in the same sense as quarks and leptons) spin-3/2 particles and of possible spin-2 "elementary" particles other than the graviton is therefore an important task. Similarly, the possible existence of "elementary" particles with spin larger than 2 cannot be excluded and deserves close theoretical and experimental study, including searches at accelerator experiments. High-spin elementary particle fields have been considered in a different approach, intermediate between SQFT and supersymmetry $[82,83]$. Then, the gravitational interaction of high spins turns out to be nonanalytic in the cosmological constant.

An alternative to SQFT where the basic vacuum structure is not dominated by the usual field condensates and zero modes has independently been suggested in $[80,84]$ and in $[23,36]$ in order to solve the standard cosmological constant problem.

The spinorial wave function of a spin-1/2 particle can feel the PSD if it is able to identify the spinorial cosmic time direction. Then, the PSD corresponds to the points on $S^{3}$ whose spinorial distance to the particle position is equal to the same spinorial time direction multiplied by a complex number. The situation may be similar for particles with other spins.

In spite of its important implications, the above described SST does not yet incorporate space units, standard matter or even a definite vacuum structure. As previously considered, the size of the SST-based universe can be much larger than that of the conventional one of present standard cosmological models and it may even happen that standard matter occupies only a small part of the SST or that its nucleation has occurred in many independent regions. Inserting standard matter in the SST would require introducing a distance unit in the spinorial $S^{3}$ constant-time hypersphere. This would simultaneously lead to a potential comparison between the size of our region of the Universe 
and that of the Universe as a whole. The cosmic SST space curvature is then expected to be much smaller than the one estimated from recent observations using the $\Lambda$ CDM approach [23, 24].

From a dynamical point of view, it seems normal to assume that the SST geometry is somehow in quasi-equilibrium with a deep underlying physical vacuum structure that can be common to all local vacua. The SST and the geometrically time-dependent radius of the Universe would then reflect by themselves the existence of dominant cosmic forces leading to this evolution in time. Such a permanent expansion of the physical vacuum suggests, in our time language, a fundamental instability that may have been at the origin of a pre-Big Bang era followed by the nucleation of standard matter in our part of the Universe. As just stressed, the notion of time itself deserves further thought [86].

\section{Pre-Big Bang}

The original (1931) Big Bang hypothesis [87] was based on Quantum Mechanics (then a recent new theory), assuming that the energy of the Universe was initially concentrated in an initial quantum. The notions of space and time were assumed to be meaningless at this stage, so that "the beginning of the world happened a little before the beginning of space and time".

But our present understanding of Physics and of its theoretical uncertainties allows to go beyond Planck scale if Quantum Mechanics is assumed to be no longer an exact law of Physics at this scale $[36,88]$. At the Planck scale, and even at a lower energy scale $E_{\text {trans }}$, standard physics may start being replaced by new physics as the dominant dynamics [33, 92], and similarly above some distance scale that can be larger than the Planck length $[24,41]$. The Planck scale does not necessarily make sense [41].

Then, a pre-Big Bang scenario can replace standard cosmology below Planck time and even below a larger time scale (the transition time $t_{\text {trans }}$ ), in such a way that the origin of time $t=0$ makes sense and becomes the beginning of a cosmological era. The SST and the superbradyon hypothesis [32, 33] can provide a useful framework for such scenarios, and similarly the kind of approach developed in $[39,40]$.

Pre-Big Bang scenarios based on strings have often been considered [89, 90], but strings are known [91] to have an underlying composite structure. Thus, using strings to describe standard "elementary" particles amounts to introducing an implicit preonic pattern $[36,88])$.

More generally, new physics including ultimate constituents of matter and new space-time properties may indeed have led the initial evolution of the Universe reaching, before conventional matter becomes apparently dominant in our region, an age and a global size that cannot be accounted for by the conventional cosmology based on Big Bang and inflation. The standard principles of Physics such as relativity and quantum mechanics are not expected to be valid in the pre-Big Bang era [81, 85]. Then, General Relativity would not be the appropriate framework to understand and describe the birth of the Universe, the structure of vacuum and their cosmological implications.

Cosmologies with a Pre-Big Bang era, including those based on the SST, can naturally avoid the basic problems of the standard Big Bang that led to the development of cosmic inflation models.

Going beyond quantum mechanics eliminates problems related to Planck scale. The global Universe can be much larger than the Planck distance at Planck time with no horizon problem and contain a very large amount of energy. Then, it is even not obvious that the standard Planck scale corresponds to any dynamical reality, and similarly for the Grand Unification scale if new physics is at work at the usually associated energy [41, 92].

As low-energy symmetries do not necessarily become more exact at very high energy $[36,97]$ the grand unification epoch can disappear in its standard form, together with the monopole problem. New Friedmann-like equations [24, 25] can automatically solve the flatness problem and do not require 
dark energy or the standard cosmological constant. Writing for the age of the Universe:

$$
t=t_{P B B}+t_{E}
$$

where $t_{P B B}$ is the time during which the pre-Big Bang dynamics dominates and $t_{E}$ corresponds to the subsequent evolution in our side of the Universe, $t_{P B B}$ can be much larger than the time scale associated to cosmic inflation in conventional cosmological models. Remnants can survive far beyond $t_{P B B}$.

As previously stressed, pre-Big Bang approaches can produce primordial gravitational waves without any need for cosmic inflation. An explicit example was provided in $[39,40]$ with an initial singularity characterized by a gravitational instanton at $t=0$. Then, CMB $B$-modes can result from primordial gravitational waves generated by the evolution of such an initial object.

The kind of basic mechanism considered in [39, 40] (an instanton-like configuration at cosmic time $t=0$ ) is a powerful one and may also allow for several kinds of original cosmological approaches besides the model explicitly developed by the authors. An initial preonic instanton, for instance, would generate preonic waves that can later give rise to gravitational waves.

Similarly, the situation for vector perturbations in pre-Big Bang Models can be substantially different from the standard approach $[17,18]$. Not only in the case of SST-based cosmologies as explained above, but more generally. In standard cosmology, vector perturbations are ignored as a possible source of $B$-modes in the primordial cosmic microwave background polarization, arguing that they would be associated to vorticity in the plasma and quickly damped by inflation [31,93]. But these considerations do not apply to most pre-Big Bang models where vector perturbations can be present already at an early stage, remain and develop in the absence of the standard inflationary evolution.

Pre-Big Bang scenarios remain a very open field where much work remains to be done with explicit formulations and tests, and that no astrophysical information invalidates at the present stage. The situation is similar concerning the possible ultimate constituents of matter.

\section{The superbradyon hypothesis}

If the vacuum is made of a fundamental matter or pre-matter different from standard matter and of which the conventional "elementary" particles are actually composite, the speed of light is not expected to be a fundamental critical speed.

The ultimate constituents of matter can have a critical speed much faster than that of light just as the speed of light is much faster than that of sound [32, 33]. As mechanisms of total confinement would not necessarily be present, it is not excluded that the ultimate fundamental objects (such as superbradyons [76]) can still exist in our Universe as free superluminal particles and be detectable even if they interact very weakly with standard matter. They can be remnants from the early Universe forming a sea of particles with speeds close to $c[34,36]$, and part of the dark matter $[81,84]$.

Superbradyons (superluminal preons) provide a simple explicit example of new ingredients that alternative cosmologies can naturally incorporate in pre-Big Bang scenarios. They lead to a new definition of causality. As in models of Lorentz symmetry violation [33, 92], the existence of a privileged rest frame (the vacuum rest frame, VRF) for each comoving observer is naturally assumed $[34,76]$. Such a hypothesis is compatible with the intrinsic SST geometry.

Superbradyons can be the basic constituents of the fundamental vacuum tacitly present in the SST approach, or be the basic ingredient of another pre-Big Bang pattern.

In a limit where the usual kinematical concepts would still make sense for such objects, a simple choice for the relation between their energy $\left(E_{s}\right)$, momentum $\left(p_{s}\right)$ and velocity $\left(v_{s}\right)$ would be [76]:

$$
\begin{array}{r}
E_{s}=c_{s}\left(p_{s}^{2}+m_{s}^{2} c_{s}^{2}\right)^{1 / 2} \\
p_{s}=m_{s} v_{s}\left(1-v_{s}^{2} c_{s}^{-2}\right)^{-1 / 2}
\end{array}
$$


where $m_{s}$ is the superbradyon mass and $c_{s}$ its critical speed assumed to be much larger than the speed of light $c$, just as $c$ is about a million times the speed of sound. $c_{s}$ defines the new causality.

Free superbradyons, if they exist, are usually assumed to have in most cases very weak direct interactions with laboratory conventional matter. The very small distance scales involved in their confinement inside standard particles, together with strong confinement forces, can be at the origin of this property. But superbradyons can play an important role in vacuum structure. When traveling at a speed larger than $c$, free superbradyons can spontaneously emit "Cherenkov" radiation in the form of standard particles [76]. Superbradyons searches at accelerators are worth considering [19].

If the ultimate constituents of matter can travel at a speed much faster than $c$ and the vacuum can expand similarly, the very early Universe is expected to have naturally undergone a correspondingly fast expansion while preserving correlations between distant points. Then, the horizon problem disappears and there is no need for inflation $[33,76]$. The superbradyon hypothesis is just an illustration of the new physics that may be present in pre-Big Bang cosmologies and provide a natural alternative, or a dynamical origin, to approaches such as those based on a varying speed of light [94, 95].

Furthermore, in the case of the SST the expansion of the Universe basically follows an intrinsic geometric law $\left(H=t^{-1}\right)$, potentially perturbed by matter interactions [24, 25]. This law is generated without any specific space variable, the cosmic time providing the only effective space scale [35, 37]. The comparison between the velocity of the Universe expansion and any critical speed of matter or pre-matter becomes possible only when matter and its constituents are explicitly introduced in the SST picture. In the presence of explicit distance units associated to matter properties, the Universe can actually be very large and expand at large scales very quickly as compared to the critical speed of any known or postulated form of matter or pre-matter, even if superbradyons can still solve the horizon problem in our region of the Universe.

The origin and the physical and cosmological meaning of the SST geometry will in any case be fundamental questions, including the sense of the notion of time and the precise nature of the mechanism that generates the SST expansion.

\section{The formation of standard matter}

The kind of scenario just described (pre-Big Bang with the SST or with another fundamental spacetime) provides natural alternatives to the standard mechanisms of the Big Bang + inflation $+\Lambda \mathrm{CDM}$ cosmology. It can, in particular, incorporate a fast enough expansion of the early Universe free of any horizon problem $[32,33]$ and leading to a new evolution pattern. Then, the formation of standard matter with its specific laws of Physics is expected to have implied a strong phase transition. Inside the early standard matter Universe, the effective vacuum structure and the basic parameters of the conventional laws of Physics may have naturally exhibited a significant time-dependence [18].

Cosmologies with a variable speed of light in the early Universe [94, 95], and the structure introduced in recent modified gravity models [96], may actually be a way to mimic some features of such a pre-Big Bang transition and of the related unconventional history of the Universe.

A link can also naturally exist between Quantum Mechanics and a superbradyonic vacuum structure. In particular, Quantum Mechanics can be an expression of the geometric SST structure in a more fundamental vacuum than that of SQFT [19]. Superbradyons can then be the constituents of such a vacuum where standard particles would be excitations of the fundamental state.

The standard cosmological constant and its usual phenomenological role are not required in such alternative cosmologies [24, 85]. The situation is similar for dark energy. We even do not necessarily expect the standard boson fields and harmonic-oscillator zero modes to be permanently condensed in vacuum in the absence of surrounding standard matter. Instead, the preonic vacuum structure can 
temporarily generate a suitable part of the usual condensates to react to the presence of conventional particles $[23,80]$. The formation of standard matter may just have been the emergence of vacuum excitations similar to phonons, solitons... $[33,76]$ in our region of the Universe without really changing the basic pre-existing vacuum structure.

The LLH law $H t=1$ automatically generated by the SST geometry is expected to have a deeper origin than in standard cosmology [25]. Contrary to conventional schemes, the global geometric curvature term from the SST will play a leading role in the relevant modified Friedmann-like equations for the conventional matter Universe [17, 24]. In the presence of matter, the $H t=1$ law can remain asymptotically valid in the large $t$ limit $[24,25]$.

Contrary to the usual dark energy hypothesis, the present acceleration of the expansion of the Universe can then be just the reflect of a fluctuation due to the early Universe dynamics producing remnant effects that vanish when $t \rightarrow \infty$ and the matter density tends to zero.

In spite of its intrinsic positive space curvature, the SST can actually describe apparent curvatures of both signs in the Universe as seen by conventional matter [24, 37]. Then, the geometric $H t=1$ law remains valid in the absence of matter irrespectively of the apparent space curvature, and can be a suitable asymptotic limit for large $t$ in all cases.

If standard matter nucleates in the early Universe inside an already expanding world with a preexisting fundamental matter or pre-matter, fluctuations allowing for galaxy formation are a natural phenomenon in such pre-Big Bang approaches. A simple scenario would be to assume that standard matter is formed through many nucleation points associated to local type I phase transitions.

Then, the existence of a local privileged space direction from the SST can manifest itself leading initially to rotational modes around this direction for each nucleation center and, subsequently, to an associated $\mathrm{CMB}$ polarization incorporating $B$-modes. In such a process, latent heat can help to generate more standard matter. But the global expansion of the Universe is a pre-existing phenomenon led by the SST geometry and not by conventional matter, even if the energy released by a type I phase transition associated to the formation of standard matter can be at the origin of local expansion effects for the conventional matter Universe.

Contrary to the standard inflationary pattern, pre-Big Bang cosmologies do not need the Universe to be isotropic as seen by a comoving observer $[21,37]$. The spinorial space-time provides an explicit example of a different scenario [23, 36] using the original cosmic coordinates.

The existence of a privileged rest frame for each comoving observer does not prevent standard relativity from remaining valid to a very good approximation, in our part of the Universe, for a wide range of energies and distance scales [41, 92]. The situation is similar for phonons and solitons in a solid lattice, as long as parameters such as the lattice spacing can be neglected. But the possible existence of a PSD is a specific signature of the SST and its potential implications require further investigation in Cosmology and Particle Physics.

\section{A new Friedmann-like equation}

As emphasized in $[24,25]$, the SST leads to a new approach to the role of space curvature in cosmology and to a new structure for Friedmann-like equations. In particular, the leading contribution to the square of the Lundmark-Lemaitre-Hubble [23] constant $H$ is provided by a SST curvature term equal to $t^{-2}$ whose sign does not depend on the space curvature felt by standard matter [24, 37].

The conventional cosmological constant scenario can then be naturally avoided [23,81], as such a term is no longer necessary.to describe the evolution of the Universe.

In [24], the following Friedmann-like relation for the standard matter universe was considered:

$$
H^{2}=8 \pi G \rho / 3-k R^{-2} c^{2}+t^{-2}+K+\Lambda c^{2} / 3
$$


where $\rho$ is the energy density associated to standard matter, $c$ the speed of light, $k$ the curvature parameter, $R$ the present curvature distance scale of the Universe (the curvature radius, and possibly the radius of the Universe, for $k=1$ ) and $\Lambda$ a possible new version of the cosmological constant decreasing like the matter density as the Universe expands. $\Lambda$ is now free of any cosmological constant problem. The new term $t^{-2}$, of cosmic geometric origin as suggested by the SST structure and the $H t=1$ law in the absence of matter, has a positive sign independent of $k$ and dominates the large scale expansion of the Universe. $K$ is a correction term accounting in particular for:

- a possible small difference between the comoving frames of standard cosmology and those (preexisting) obtained from the underlying SST cosmic geometry;

- similarly, a correction related to remnant effects from the pre-Big Bang era;

- a reaction of the nucleated standard matter to the pre-existing expansion of the Universe led by the SST geometry and the pre-Big Bang vacuum [23, 25];

- vacuum inhomogeneities at cosmic scale and other non-standard effects.

In spite of the existence of a local PSD, the LLH law $H t=1$ has a clearly isotropic nature, and so is equation (7). To reach equation (7), we noticed that, contrary to General Relativity, the SST curvature does not vanish in the absence of standard matter and of a cosmological constant. This situation requires a modification of the Einstein field equation [98, 99]:

$$
R_{\mu \nu}-1 / 2 g_{\mu \nu}+g_{\mu v} \Lambda=8 \pi G c^{-4} T_{\mu \nu}
$$

$\left(R_{\mu \nu}=\right.$ Ricci curvature tensor, $g_{\mu \nu}=$ metric tensor, $T_{\mu \nu}=$ stress-energy tensor $)$ in order to account for the pre-existing global cosmic curvature of the SST.

One can then write and adapted version of (8):

$$
R_{\mu \nu}-1 / 2 g_{\mu \nu}+g_{\mu \nu}^{\prime} S+g_{\mu \nu} \Lambda=8 \pi G c^{-4} T_{\mu \nu}
$$

where the term $g_{\mu \nu}^{\prime} S$ describes the SST curvature term.

In a frame where $g_{\mu \nu}^{\prime} S$ is diagonal, the structure of $g_{\mu \nu}^{\prime} S$ is fundamentally different from standard relativity. The speed of light $c$ does not govern the ratio between time and space components, so that a very small SST space curvature is compatible with a dominant time-like contribution from the same $g_{\mu \nu}^{\prime} S$ tensor to equation (7). The term $t^{-2}$ in (7) is generated in this way [23, 24] and explicitly associated to a strong violation of General Relativity by the dominant SST cosmic geometry.

Similar steps can be taken to account for the role of the PSD, considering the standard RobertsonWalker equation [100] together with (8) and (9) and introducing modifications to account for the local space anisotropy associated to the privileged direction. One can then expect to obtain: i) possible new changes in (7), basically through a further modification of $K$ and $\Lambda$; ii) a new, separate equation describing the anisotropic implications of the PSD [19]. Equation (7) tends asymptotically to the $H t=1$ law for $t \rightarrow \infty$ if $\rho, K$ and $\Lambda$ vanish in this limit [24, 25]. This is a significant difference with respect to standard cosmology, even if new quantum gravity models are being considered using nonlocality as a cutoff [101]. The $t^{-2}$ term in (7) has a deeper origin than gravity and field theory.

A further discussion of some of these questions is presented in [18]

\section{Ultra-high energy cosmic rays}

Fundamental physics at ultra-high energy (UHE), including possible effects of new physics, remains poorly known [41, 92] and requires further experimental and theoretical effort.

It is even not yet clear $[102,103]$ if the observed fall of the ultra-high energy cosmic-ray (UHECR) spectrum is a signature of the Greisen-Zatsepin-Kuzmin (GZK) cutoff or corresponds to the maximum 
energies available at astrophysical sources. Such a limitation makes difficult to interpret data [104] on UHECR traveling on moderate extragalactic distances.

Similarly, there is by now no real proof of the validity of models and algorithms for the highest energies used in data analysis to study UHECR interactions.

Exploring as far as possible the properties of UHECR, searching in particular for signatures of new physics, is an important task for a better understanding of the basic physics involved in the early Universe dynamics. Systematic tests of Lorentz symmetry at UHE [33, 34] should be pursued, together with any feasible check of other fundamental principles of standard physics [36, 105].

Information on the validity of standard low-energy symmetries at very high energy would also be of the highest interest [92,97], ad similarly for particle propagation in vacuum.

Another important issue, in connection with the subject dealt with here, is whether cosmic rays at very high energy can be sensitive to the privileged space direction generated by the SST. In particular, possible correlations between data on high-energy cosmic rays and the recent Planck observation of CMB anisotropies [20] deserve a long-term systematic exploration.

New particles can also exist as high-energy cosmic rays resulting from the decay of unconventional heavy objects (e.g. superbradyons) $[34,106]$. If superbradyons (possibly part of the dark matter) have very large rest energies, they can annihilate or decay producing standard or lower-mass superbradyonic particles with very high energies and possibly able to reach cosmic ray detectors. The search for direct superbradyon signatures in cosmic ray experiments or at accelerators must take into account the possible very weak interaction rate, as well as the violations of standard causality that may result from superluminal propagation [35].

More details on some of these topics can be found in [19].

\section{Conclusion and comments}

The possible existence of CMB B-modes of primordial origin, initially claimed on the grounds of BICEP2 data but not confirmed by more recent analyses, would in any case not be a compelling evidence for the standard Big Bang model with cosmic inflation.

Instead, such a signal, if ever really found, can be one of the natural signatures of a pre-Big Bang era and of a spinorial space-time geometry. Then, primordial vector perturbations would be an alternative to gravitational waves to generate CMB B-modes, even if primordial gravitational waves can also be present. Existing and future experiments and observations will hopefully help to answer the present fundamental interrogations of Cosmology and Particle Physics.

Before the BICEP2 announcement, Planck results on CMB anisotropy with a possible local privileged space direction combined with parity violation [20] had already suggested that nonstandard cosmological phenomena may be at work. Together with these Planck data, the discovery of primordial CMB $B$-modes would potentially provide an unprecedented evidence for physics beyond the standard model and for a space-time (the SST) acting at a deeper level than that of conventional relativity.

A new cosmology would then replace the pattern based on $\Lambda \mathrm{CDM}$ and inflation. In the presence of Pre-Big Bang scenarios, cosmic inflation is not required and looks even artificial, as the pre-Big Bang models considered here already incorporate a phase transition associated to the formation of standard matter from ultimate constituents in a fundamental vacuum different from that of SQFT. Similarly, the SST appears as a natural space-time for a world with fermions.

Not only pre-Big Bang scenarios can naturally generate primordial vector perturbations producing CMB $B$-modes, as well as gravitational waves leading to the same effect, but the standard cosmological constant is no longer required $[23,24]$ and the $H t=1$ law can be a suitable asymptotic limit at large $t$ as suggested by the SST geometry [24, 25]. 
Cosmological tests and data analyses by experimental collaborations remain by now restricted a priori to the $\Lambda \mathrm{CDM}$ dogma and devoted to the search for evidences confirming the Big Bang + inflation scenario. This is a serious limitation and a possible source of bias, as alternative cosmologies cannot be excluded and may already have been confirmed by observed signatures.

Prospects of new physics are further discussed in [19], including complementary material on the subjects dealt with here and on the possible origin of Quantum Mechanics.

\section{References}

[1] BICEP2 Collaboration, Detection Of B-mode Polarization at Degree Angular Scales by BICEP2, Physical Review Letters 112, 241101 (June 2014). Original preprint version (March 2014): arXiv:1403.3985v1.

[2] BICEP2 Collaboration, BICEP2 II: Experiment and Three-Year Data Set, arXiv:1403.4302.

[3] A. Iljjas, P.J. Steinhardt and A. Loeb, Inflationary paradigm in trouble after Planck2013, arXiv:1402.6980, and references therein.

[4] A. Iljjas, P.J. Steinhardt and A. Loeb, Inflationary schism after Planck2013, Phys.Lett.B 723, 261 (2013), arXiv:1304.2785, and references therein.

[5] A.H. Guth, D.I. Kaiser and Y. Nomura, Inflationary paradigm after Planck 2013, arXiv: 1312.7619, and references therein.

[6] A. Linde, Inflationary Cosmology after Planck 2013, arXiv:1402.0526, and references therein.

[7] Planck mission, European Space Agency, http://sci.esa.int/planck/

[8] The Planck Collaboration, Planck 2013 results. XVI. Cosmological parameters, arXiv:1303.5076.

[9] The Planck Collaboration, Planck 2013 results. XXII. Constraints on inflation, arXiv:1303.5082.

[10] The Planck Collaboration, Planck intermediate results. XXX. The angular power spectrum of polarized dust emission at intermediate and high Galactic latitudes, arXiv:1409.5738.

[11] H.Liu, P. Mertsch and S. Sarkar, Fingerprints of Galactic Loop I on the Cosmic Microwave Background, arXiv:1404.1899

[12] See also the Planck Collaboration, Planck intermediate results. XIX. An overview of the polarized thermal emission from Galactic dust, arXiv:1405.0871, and subsequent papers. arXiv:1405.0872, arXiv:1405.0873 and arXiv:1405.0874.

[13] See, for instance, ESA and Planck, Planck: gravitational waves remain elusive, http://sci.esa.int/planck/55362-planck-gravitational-waves-remain-elusive/

[14] BICEP2/Keck and Planck Collaborations, A joint analysis of BICEP2/Keck Array and Planck data, arXiv:1502.00612.

[15] M.J. Mortonson and U. Seljak, A joint analysis of Planck and BICEP2 B modes including dust polarization uncertainty, arXiv:1405.5857.

[16] R. Flauger, J. C. Hill and D. N. Spergel, Toward an Understanding of Foreground Emission in the BICEP2 Region, arXiv:1405.7351.

[17] L. Gonzalez-Mestres, CMB B-modes, spinorial space-time and Pre-Big Bang (I), mp_arc 14-16, and references therein.

[18] L. Gonzalez-Mestres, CMB B-modes, spinorial space-time and Pre-Big Bang (II), mp_arc 1460 , and references therein.

[19] L. Gonzalez-Mestres, Tests and prospects of new physics at very high energy, these Proceedings.

[20] The Planck Collaboration, Planck 2013 results. XXIII. Isotropy and statistics of the CMB, arXiv:1303.5083 and references therein. 
[21] L. Gonzalez-Mestres, Spinorial space-time and privileged space direction (I), mp_arc 13-75, and references therein.

[22] See, for instance, The Kavli Foundation, A New Baby Picture of the Universe, http://www.kavlifoundation.org/science-spotlights/kicc-planck-universe

[23] L. Gonzalez-Mestres, Pre-Big Bang, fundamental Physics and noncyclic cosmologies, International Conference on New Frontiers in Physics, ICFP 2012, Kolymbari, Crete, June 10-16 2012, EPJ Web of Conferences 70, 00035 (2014), and references therein. Preprint at mp_arc 13-18.

[24] L. Gonzalez-Mestres, Pre-Big Bang, space-time structure, asymptotic Universe, 2nd International Conference on New Frontiers in Physics, Kolymbari, Crete, Greece, August 28 - September 5, 2013, EPJ Web of Conferences 71, 00063 (2014), references therein and Post Scriptum to the preprint hal-00983005.

[25] L. Gonzalez-Mestres, Planck data, spinorial space-time and asymptotic Universe, mp_arc 1333, and references therein.

[26] A. Ijjas, J.-L. Lehners and P.J. Steinhardt, Phys. Rev. D 89, 123520 (2014), arXiv:1404.1265.

[27] R. Kallosh, A. Linde and A. Westphal, Chaotic Inflation in Supergravity after Planck and BICEP2, Phys. Rev. D 90, 023534 (2014), arXiv:1405.0270.

[28] P.J. Steinhardt, Big Bang blunder bursts the multiverse bubble, Nature 510, 9 (2014).

[29] P.J. Steinhardt, The inflation debate, Scientific American, April 2011, 36, http://www.physics.princeton.edu/ steinh/0411036.pdf

[30] G.W. Gibbons and N. Turok, The Measure Problem in Cosmology, Phys.Rev.D 77, 063516 (2008), arXiv:hep-th/0609095.

[31] See, for instance, W. Hu and M. White, A CMB Polarization Primer, New Astron. 2, 323 (1997), arXiv:astro-ph/9706147, and references therein.

[32] L. Gonzalez-Mestres, Cosmological Implications of a Possible Class of Particles Able to Travel Faster than Light, Proceedings of the TAUP 1995 Conference, Nucl. Phys. Proc. Suppl. 48 (1996), 131, arXiv:astro-ph/9601090.

[33] L. Gonzalez-Mestres, Vacuum Structure, Lorentz Symmetry and Superluminal Particles, arXiv:physics/9704017.

[34] L. Gonzalez-Mestres, Physical and Cosmological Implications of a Possible Class of Particles Able to Travel Faster than Light, contribution to the 28th International Conference on High Energy Physics, Warsaw 1996, arXiv:hep-ph/9610474, and references therein.

[35] L. Gonzalez-Mestres, Space, Time and Superluminal Particles, arXiv:physics/9702026.

[36] L. Gonzalez-Mestres, Cosmic rays and tests of fundamental principles, CRIS 2010 Proceedings, Nucl. Phys. B, Proc. Suppl. 212-213 (2011), 26, and references therein. The arXiv.org version arXiv:1011.4889 includes a relevant Post Scriptum.

[37] L. Gonzalez-Mestres, Spinorial space-time and Friedmann-like equations (I), mp_arc 13-80, and references therein.

[38] Wilkinson Microwave Anisotropy Probe, http://map.gsfc.nasa.gov/

[39] G. Bogdanoff, Fluctuations quantiques de la signature de la métrique à l'échelle de Planck, Thesis, Université de Bourgogne 1999, and related published papers.

[40] I. Bogdanoff, Etat topologique de l'espace-temps à l'échelle 0, Thesis, Université de Bourgogne 2002, and related published papers.

[41] L. Gonzalez-Mestres, Ultra-high energy physics and standard basic principles, 2nd International Conference on New Frontiers in Physics, Kolymbari, Crete, Greece, August 28 - September 5, 2013, EPJ Web of Conferences 71, 00062 (2014), and Post Scriptum to the preprint mp_arc 14-31. 
[42] H. Poincaré, Sur la dynamique de l'électron, Comptes rendus de l'Académie des Sciences 140, 1504 (1905), http://smf4.emath.fr/Publications/Gazette/2005/104/smf_gazette_104_60-70.pdf

[43] A. Einstein, Geometrie und erfahrung, Preus. Akad. der Wissench., Sitzungsberichte, part I, p. 123 (1921), http://www.philoscience.unibe.ch/documents/kursarchiv/WS99/Geometrie.pdf . English translation Geometry and experience in Sidelights on relativity, Methuen, London 1922, http://www.gutenberg.org/files/7333/7333-h/7333-h.htm

[44] Caltech Observational Cosmology Group, BICEP: Robinson Gravitational Wave Background Telescope, http://bicep.caltech.edu/public/

[45] K.W. Yoon et al., The Robinson Gravitational Wave Background Telescope (BICEP): a bolometric large angular scale CMB polarimeter, in Millimeter and Submillimeter Detectors and Instrumentation for Astronomy III, Proceedings of SPIE, Vol. 6275 (2006), arXiv:astro-ph/0606278.

[46] BICEP1 Collaboration, Degree-Scale CMB Polarization Measurements from Three Years of BICEP1 Data, ApJ 783, 67 (2014), arXiv:1310.1422.

[47] C.L. Kuo et al., Antenna-coupled TES bolometer arrays for CMB polarimetry, in SPIE Proceedings Vol. 7020, Marseille, 2008, arXiv:0908.1464.

[48] A. Orlando et al., Antenna-coupled TES Bolometer Arrays for BICEP2/Keck and SPIDER, in SPIE Proceedings Vol. 7741, San Diego, 2010, arXiv:1009.3685.

[49] C.L. Kuo et al., Antenna-coupled TES bolometers for the Keck Array, Spider, and Polar-1, arXiv: 1208.1247. See also http://www.cfa.harvard.edu/CMB/bicep2/ and http://www.cfa.harvard.edu/CMB/keckarray/

[50] J.A. Brevik et al., Initial performance of the BICEP2 antenna-coupled superconducting bolometers at the South Pole, in SPIE Proceedings Vol. 7741, San Diego, 2010, http://authors.library.caltech.edu/22834/1/Brevik2010p12867Adaptive_Optics_Systems_Pts_13.pdf

[51] Jet Propulsion Laboratory, California Institute of Technology, TES Bolometers Enable a New Probe of the Infant Universe, http://microdevices.jpl.nasa.gov/capabilities/superconductingdevices/tes-bolometers.php

[52] C.D. Sheehy et al., The Keck Array: a pulse tube cooled CMB polarimeter, arXiv:1104.5516.

[53] S. Kernasovskiy et al., Optimization and sensitivity of the Keck Array, arXiv: 1208.0857.

[54] Z.Ahmed et al., BICEP3: a $95 \mathrm{GHz}$ refracting telescope for degree-scale CMB polarization, arXiv:1407.5928.

[55] B.A. Benson et al., A Next-Generation Cosmic Microwave Background Polarization Experiment on the South Pole Telescope, arXiv:1407.2973.

[56] POLAR CMB Polarization Experiment, http://polar-array.stanford.edu/ (Stanford University). See also Chao Lin-Kuo transparencies at the 2009 Meeting of the SLAC Users Organization.

[57] R. O'Brient et al., Antenna-coupled TES bolometers for the Keck Array, Spider, and Polar-1, arXiv:1208.1247.

[58] The POLARBEAR Collaboration, A Measurement of the Cosmic Microwave Background BMode Polarization Power Spectrum at Sub-Degree Scales with POLARBEAR, Astrophysical Journal 794, 171 (2014), arXiv:1403.2369.

[59] Z. Kermish et al., The POLARBEAR Experiment, arXiv:1210.7768.

[60] D. Barron et al., Development and characterization of the readout system for POLARBEAR-2, arXiv:1410.7488.

[61] Atacama Cosmology Telescope (ACT), http://lambda.gsfc.nasa.gov/product/act/ (NASA) and http://www.princeton.edu/act/ (Princeton University). 
[62] M.D. Niemack et al., ACTPol: A polarization-sensitive receiver for the Atacama Cosmology Telescope, in SPIE Proceedings Vol. 7741, San Diego, 2010, arXiv:1006.5049.

[63] A. van Engelen, The Atacama Cosmology Telescope: Lensing of CMB Temperature and Polarization Derived from Cosmic Infrared Background Cross-Correlation, arXiv:1412.0626.

[64] J.E. Austermann et al., SPTpol: an instrument for CMB polarization measurements with the South Pole Telescope, arXiv:1210.4970.

[65] K.T. Story et al., A Measurement of the Cosmic Microwave Background Gravitational Lensing Potential from 100 Square Degrees of SPTpol Data, arXiv:1412.4760.

[66] J.W. Appel et al., The Cosmology Large Angular Scale Surveyor (CLASS): $38 \mathrm{GHz}$ detector array of bolometric polarimeters, arXiv: 1408.4789.

[67] K. Rostem et al., Scalable background-limited polarization-sensitive detectors for mm-wave applications, arXiv:1408.4790.

[68] J.P. Filippini et al., SPIDER: a balloon-borne CMB polarimeter for large angular scales, arXiv:1106.2158.

[69] A.S. Rahlin et al., Pre-flight integration and characterization of the SPIDER balloon-borne telescope, arXiv:1407.2906.

[70] R. Misawa et al., PILOT: a balloon-borne experiment to measure the polarized FIR emission of dust grains in the interstellar medium, arXiv:1410.5760.

[71] The PILOT project, http://pilot.irap.omp.eu/

[72] H.K. Eriksen et al., Asymmetries in the CMB anisotropy field, Astrophys.J. 605, 14 (2004) and Erratum ibid. 609, 1198 (2004), arXiv: astro-ph/0307507.

[73] F. K. Hansen, A. J. Banday and K. M. Gorski, Testing the cosmological principle of isotropy: local power spectrum estimates of the WMAP data, Mon.Not.Roy.Astron.Soc. 354, 641 (2004), arXiv: astro-ph/0404206.

[74] C.-G. Park, Non-Gaussian Signatures in the Temperature Fluctuation Observed by the Wilkinson Microwave Anisotropy Probe, Mon.Not.Roy.Astron.Soc. 349, 313 (2004), arXiv:astro-ph/0307469.

[75] ESA-Planck News, Planck reveals an almost perfect Universe, 21 March 2013 article.

[76] L. Gonzalez-Mestres, Properties of a possible class of particles able to travel faster than light, Proceedings of the January 1995 Moriond Workshop, Ed. Frontières, arXiv:astro-ph/9505117.

[77] K. Lundmark, Curvature of Space-Time in de Sitter's World, MNRAS 84 (1924), 747, http://articles.adsabs.harvard.edu/full/1924MNRAS..84..747L

[78] G. Lemaître, Un Univers homogène de masse constante et de rayon croissant, rendant compte de la vitesse radiale des nébuleuses extra-galactiques, Ann. Soc. Sci. Brux. A 47 (1927), 49, http://articles.adsabs.harvard.edu/full/1927ASSB...47...49L

[79] E. Hubble, A relation between distance and radial velocity among extra-galactic nebulae, PNAS 15 (1929), 168, http://www.pnas.org/content/15/3/168

[80] L. Gonzalez-Mestres, Lorentz symmetry violation, dark matter and dark energy, Proceedings of the Invisible Universe International Conference (Paris 2009), AIP Conf.Proc. 1241 (2010),120. The arXiv.org version arXiv:0912.0725 contains a relevant Post Scriptum.

[81] L. Gonzalez-Mestres, Pre-Big Bang, vacuum and noncyclic cosmologies, Europhysics Conference on High Energy Physics, Grenoble, July 2011, PoS EPS-HEP2011 479, and references therein.

[82] See, for instance, M.A. Vasil'ev and E.S. Fradkin, Gravitational interaction of massless high$\operatorname{spin}(s>2)$ fields, JETP Letters 44, 622 (1986).

[83] V.E. Didenko and E.D. Skvortsov, Elements of Vasiliev theory, arXiv:1401.2975. 
[84] L. Gonzalez-Mestres, Superbradyons and some possible dark matter signatures, arXiv:0905.4146.

[85] L. Gonzalez-Mestres, WMAP, Planck, cosmic rays and unconventional cosmologies, contribution to the Planck 2011 Conference, Paris, January 2011, arXiv:1110.6171.

[86] Some examples of recent attempts to understand the origin of space and time are quoted in $\mathrm{Z}$. Merali, Theoretical physics: The origins of space and time, Nature 500, 516 (2013).

[87] G. Lemaître, The Beginning of the World from the Point of View of Quantum Theory, Nature 127, 706 (1931).

[88] L. Gonzalez-Mestres, Preon models, relativity, quantum mechanics and cosmology (I), arXiv:0908.4070, and references therein.

[89] See, for instance, M. Gasperini and G. Veneziano, in Beyond the Big Bang : Competing Scenarios for an Eternal Universe, Ed. R. Vaas, Springer-Verlag 2007, arXiv:hep-th/0703055.

[90] M. Gasperini, String theory and primordial cosmology, arXiv:1402.0101.

[91] See, for instance, P. Aurenche and L. Gonzalez-Mestres, Glueball singularity, flavor loops and the Harari-Freund picture, Zeitschrift für Physik C 1, 307 (1979), CERN preprint TH.2568, and references therein.

[92] L. Gonzalez-Mestres, High-energy cosmic rays and tests of basic principles of Physics, International Conference on New Frontiers in Physics, ICFP 2012, Kolymbari, Crete, June 10-16 2012, EPJ Web of Conferences 70, 00047 (2014), and references therein. Preprint at mp_arc 13-19.

[93] See, for instance Y.D. Takahashi, Cosmic Microwave Background Polarization: The Next Key Toward the Origin of the Universe, Berkeley 2009, and Wonders of the Cosmos, Berkeley 2010.

[94] J.W. Moffat, Variable Speed of Light Cosmology, Primordial Fluctuations and Gravitational Waves, arXiv:1404.5567.

[95] J.W. Moffat, Superluminal Gravitational Waves, arXiv: 1406.2609.

[96] J.W. Moffat, Structure Growth and the CMB in Modified Gravity (MOG), arXiv: 1409.0853.

[97] L. Gonzalez-Mestres, Testing fundamental principles with high-energy cosmic rays, HEP Europhysics Conference, Grenoble, July 2011, PoS EPS-HEP2011 390, and references therein.

[98] A. Einstein, Die Feldgleichungen der Gravitation, Sitzungsberichte der Preussischen Akademie der Wissenschaften zu Berlin (1915), 844.

[99] A. Einstein, Die Grundlage der allgemeinen Relativitätstheorie, Annalen der Physik 354 (7) (1916), 769.

[100] See, for instance, M. Trodden and S.M. Carroll, TASI lectures: Introduction to Cosmology, arXiv: astro-ph/0401547.

[101] J.W. Moffat, Quantum Gravity and the Cosmological Constant Problem, arXiv:1407.2086

[102] The Pierre Auger Collaboration, Hightlights from the Pierre Auger Observatory, contribution to the ICRC 2013 Conference, arXiv:1310.4620, and references therein.

[103] The Pierre Auger Observatory, Contributions to the 33rd International Cosmic Ray Conference (ICRC 2013), arXiv:1307.5059, and references therein.

[104] The Telescope Array Collaboration, Indications of Intermediate-Scale Anisotropy of Cosmic Rays with Energy Greater Than 57 EeV in the Northern Sky Measured with the Surface Detector of the Telescope Array Experiment, arXiv:1404.5890.

[105] L. Gonzalez-Mestres, Preon models, relativity, quantum mechanics and cosmology (I), arXiv:0908.4070.

[106] L. Gonzalez-Mestres, Superluminal Matter and High-Energy Cosmic Rays, arXiv:astro$\mathrm{ph} / 9606054$, and references therein. 